Erratum

\title{
Reflection and transmission of optical narrow-extent pulses by axially excited chiral sculptured thin films
}

J.B. Geddes III and A. Lakhtakia

CATMAS - Computational and Theoretical Materials Sciences Group, Department of Engineering Science and Mechanics, 212 Earth-Engineering Sciences Building, Pennsylvania State University, University Park, PA 16802-6812, USA

Eur. Phys. J. AP 13, 3-14 (2001)

The same typographical error crept in the last term on the right sides of equations (19), (21) and (23) referred of the paper above. Please replace -1 by $-\delta(t)$. All other equations as well as our results are not affected. We regret any inconvenience caused by this error. 